\title{
Selective Chemical Labeling and Sequencing of 5-Hydroxymethylcytosine in DNA at Single-Base Resolution
}

\author{
Xiaogang $\mathrm{Li}^{1,2,3}$, Xinxin Shi ${ }^{4}$, Yin Gong ${ }^{5}$, Wenting Guo ${ }^{2}$, Yuanrui Liu ${ }^{6 *}$, Chunwei Peng ${ }^{4 \star}$ and \\ Yingchun $\mathrm{Xu}^{1,3 *}$
}

${ }^{1}$ Department of Clinical Laboratory, State Key Laboratory of Complex Severe and Rare Diseases, Peking Union Medical College Hospital, Chinese Academy of Medical Science and Peking Union Medical College, Beijing, China, ${ }^{2}$ Medical Research Center. State Key Laboratory of Complex Severe and Rare Diseases, Peking Union Medical College Hospital, Chinese Academy of Medical Science and Peking Union Medical College, Beijing, China, ${ }^{3}$ Beijing Key Laboratory for Mechanisms Research and Precision Diagnosis of Invasive Fungal Diseases, Beijing, China, ${ }^{4}$ Gastrointestinal Surgery Department of the First Affiliated Hospital of Anhui Medical University, Hefei, China, ${ }^{5}$ School of Electronics Engineering and Computer Science, Peking University, Beijing, China, ${ }^{6}$ Characteristic Medical Center of the Chinese People's Armed Police Force, Tianjin, China

OPEN ACCESS

Edited by:

Mojgan Rastegar,

University of Manitoba, Canada

Reviewed by:

Bi-Feng Yuan,

Wuhan University, China

Alexey Fomenkov,

New England Biolabs, United States

*Correspondence:

Yingchun $X u$

xycpumch@139.com

Chunwei Peng

pcwayfy@163.com

Yuanrui Liu

Ibekeyl@qq.com

Specialty section:

This article was submitted to Epigenomics and Epigenetics,

a section of the journal

Frontiers in Genetics

Received: 29 July 2021 Accepted: 08 October 2021

Published: 17 November 2021

Citation:

Li X, Shi X, Gong Y, Guo W, Liu Y,

Peng $C$ and $X u Y(2021)$ Selective

Chemical Labeling and Sequencing of

5-Hydroxymethylcytosine in DNA at

Single-Base Resolution.

Front. Genet. 12:749211.

doi: 10.3389/fgene.2021.749211
5-Hydroxymethylcytosine (5hmC), the oxidative product of 5-methylcytosine $(5 \mathrm{mC})$ catalyzed by ten-eleven translocation enzymes, plays an important role in many biological processes as an epigenetic mediator. Prior studies have shown that $5 \mathrm{hmC}$ can be selectively labeled with chemically modified glucose moieties and enriched using click chemistry with biotin affinity approaches. Besides, DNA deaminases of the AID/APOBEC family can discriminate modified $5 \mathrm{hmC}$ bases from cytosine $(\mathrm{C})$ or $5 \mathrm{mC}$. Herein, we developed a method based on embryonic stem cell (ESC) whole-genome analysis, which could enrich 5hmC-containing DNA by selective chemical labeling and locate $5 \mathrm{hmC}$ sites at single-base resolution with enzymebased deamination. The combination experimental design is an extension of previous methods, and we hope that this cost-effective single-base resolution $5 \mathrm{hmC}$ sequencing method could be used to promote the mechanism and diagnosis research of $5 \mathrm{hmC}$.

Keywords: 5-hydroxymethylcytosine, single-base resolution, chemical labeling, cost-effective, AID/APOBEC family

\section{INTRODUCTION}

5-Hydroxymethylcytosine $(5 \mathrm{hmC})$, which exists in various mammalian tissues and cell types, is an oxidative product of 5-methylcytosine $(5 \mathrm{mC})$ catalyzed by ten-eleven translocation (TET) enzymes (Kriaucionis and Heintz, 2009; Tahiliani et al., 2009). Many researches show that $5 \mathrm{hmC}$ not only is an intermediate of DNA demethylation but also plays an important role in many biological processes and human diseases as an epigenetic mediator (Szulwach et al., 2011; Mellén et al., 2012; Colquitt et al., 2013; Taylor et al., 2016). The recent development of high-throughput sequencing technology has enabled whole-genome sequencing of $5 \mathrm{hmC}$ in mammalian systems. Generally, there are two strategies, which are the selective enrichment-based profiling and deamination-based single-base resolution sequencing methods (Song et al., 2011; Pastor et al., 2012; Tan et al., 2013; Thomson et al., 2013; Cui et al., 2014; Petterson et al., 2014; Sun et al., 2015; Han et al., 2016; Hu et al., 2019; Liu et al., 2019; Gibas et al., 2020; Wang et al., 2021). While the current application of these methods provides key information about the distribution and functional insights of $5 \mathrm{hmC}$, there is major shortage for both strategies. The lack of single-base resolution information of the profiling strategy limited its application in detailed $5 \mathrm{hmC}$ 
location context, while the single-base resolution method is limited by its high sequencing cost. Therefore, a method that has both advantage of single-base resolution and enrichment would be highly valued in broad biological and clinical studies. A recent study developed a bisulfite-free strategy to detect $5 \mathrm{hmC}$ in single-base resolution using AID/APOBEC family DNA deaminase enzyme as deamination reagent, in which $5 \mathrm{hmC}$ is protected by a glucose motif catalyzed by T4 beta-glucosyltransferase (T4- $\beta$ GT) (Liu et al., 2019; Vaisvila et al., 2019; Sun et al., 2021a). This strategy has also been reported in a well-studied chemical selective profiling method back to year 2011 using a modified UDP-glucose (Song et al., 2011).

Here, we introduce a cost-effective single-base resolution $5 \mathrm{hmC}$ sequencing method, which allows genome-wide chemical labeling and enrichment of $5 \mathrm{hmC}$ and a bisulfite-free single-base resolution detection of $5 \mathrm{hmC}$. Our strategy (DIP-CAB-Seq) has three steps: 1) label and enrich the $5 \mathrm{hmC}$ containing fragments using the $\mathrm{N}_{3^{-}}$ UDP-glucose, click chemistry, and biotin-avidin interaction; 2) deaminate unprotected cytosines and $5 \mathrm{mC}$ using a deaminase; and 3) construct sequencing library in a single-stranded manner. This strategy is compatible with the labeling chemistry, enzyme-based deamination, and single-stranded DNA library construction. To demonstrate the superiority and effectiveness of this method, we have applied this approach to compare detected $5 \mathrm{hmC}$ signals among our new methods (DIP-CAB-Seq), 5hmC-Seal and ACESeq (Song et al., 2011; Liu et al., 2019). We found that we can detect reliable single-base $5 \mathrm{hmC}$ signals using DIP-CAB-Seq method with limited sequencing depth.

\section{METHODS}

\section{Cell Culture and DNA Preparation}

The v6.5 mouse embryonic stem cells (mESCs) are the same cell line from a previous study (Wray et al., 2011; Guo et al., 2021). Cell culture and genomic DNA was prepared as previously described. Briefly, mESCs were cultured on $0.1 \%$ gelatin-coated plates in $2 \mathrm{i}+$ leukemia inhibitory factor $(2 \mathrm{i}+\mathrm{LIF})$ media, which consist of N2B27 (DMEM)/F-12, neurobasal, N-2 supplement, and B27 supplement, supplemented with $1 \mu \mathrm{mol} / \mathrm{L}$ of PD0325901 (PZ0162, SigmaAldrich, St. Louis, MO, USA), $3 \mu \mathrm{mol} / \mathrm{L}$ of CHIR99021 (SML1046, Sigma-Aldrich), and 1,000 U/ml LIF (PMC9484; Gibco, Grand Island, NY, USA). The genomic DNA was extracted by sodium dodecyl sulfate (SDS)/proteinase K digestion, phenol/chloroform extraction, and ethanol precipitation.

\section{Library Construction}

\section{Nano-ACE-Seq Library Construction of 5-Hydroxymethylcytosine-Containing Genomic DNA (DIP-CAB-Seq)}

The mESC genomic DNA was sheared with KAPA Frag Kit (KK8600, Kapa Biosystems, Wilmington, MA, USA) to average 200 base pair and purified using the manufacturer's protocol (Ring et al., 2017). Fragmented mESC genomic DNA measuring $500 \mathrm{ng}$ (average $200 \mathrm{bp}$ ) was treated with $\beta$-GT (New England Biolabs, Ipswich, MA, USA; Catalog \#M0357S) in the presence of UDP-6$\mathrm{N}_{3}$-Glu and labeled with cyclooctyne-biotin. Subsequently, 5hmCcontaining fragments were enriched using Streptavidin beads. The enriched fragments were mixed with control DNAs, CPG methylated pUC19, and unmethylated lambda and subjected to denaturation with $\mathrm{NaOH}$ and enzymatic deamination using APOBEC enzyme (New England Biolabs, Catalog \#E7120S) to transform $\mathrm{C} / \mathrm{mC}$ to $\mathrm{U}$ but not hmC. Then the converted DNA was tagged with Illumina compatible adapter and amplified to an appropriate library concentration using Accel-NGS Methyl-Seq DNA Library Kit (Swift BioSciences, Ann Arbor, MI, USA) combined with NEB Next Multiplex Oligos for Illumina. Libraries were checked for quality and quantified using Agarose Gel Electrophoresis and Qubit3.0 individually.

\section{5-Hydroxymethylcytosine-Seal Library Construction of 5-Hydroxymethylcytosine-Containing Genomic DNA}

$5 \mathrm{hmC}$-Seal Library was prepared as previously described (Song et al., 2011). Briefly, the fragmented mESC genomic DNA (average $200 \mathrm{bp}$ ) was treated with UDP-6- $\mathrm{N}_{3}$-Glu in the presence of $\beta$-GT to form chemical modification, followed by labeling with $\mathrm{DBCO}-\mathrm{PEG}_{4}$-Biotin via click reaction. The $5 \mathrm{hmC}$ containing DNA fragments were captured by $\mathrm{C} 1$ Streptavidin beads. The beads with enriched DNA fragments were resuspended in water and amplified with 12-17 cycles of PCR using an enzyme mixture in the Nextera kit. The PCR products were purified using AMPure XP beads. Sequencing was performed on the NextSeq instrument.

\section{ACE-Seq Library Construction of} 5-Hydroxymethylcytosine-Containing Genomic DNA ACE-Seq Library was prepared as previously described (Liu et al., 2019). The mESC genomic DNA was sheared with KAPA Frag Kit (KK8600, Kapa Biosystems, Wilmington, MA, USA) to an average of 200 base pair and purified using the manufacturer's protocol (Ring et al., 2017). DNA fragment was prepared to a concentration of $15-20 \mathrm{ng} / \mu \mathrm{l}$. The reaction was assembled in a total volume of $50 \mu \mathrm{l}$ using the table below as per reaction. If multiple samples were processed, making a master mix of everything except the sample should be recommended.

The reaction was placed in a thermocycler and incubated at $37^{\circ} \mathrm{C}$ for $1 \mathrm{~h}$. Subsequent reaction was cleaned separately by DNA Clean \& Concentrator-5 Kit (Zymo Research, Irvine, CA, USA) and eluted in $25 \mu \mathrm{l}$ of EB buffer. Glycosylated dsDNA was treated with a fresh $0.1 \mathrm{~N} \mathrm{NaOH}$ solution and incubated in a thermocycler at $50^{\circ} \mathrm{C}$ for $10 \mathrm{~min}$ for denaturation. After that, the sample was transferred into an ice box and was left to stand at least $5 \mathrm{~min}$ to keep the DNA in its single-stranded form. Subsequently, the ssDNA was subjected to enzymatic deamination using APOBEC enzyme to transform $\mathrm{C} / \mathrm{mC}$ to $\mathrm{U}$ but not hmC with NEB Next ${ }^{\circledR}$ Enzymatic Methyl-seq Kit (New England Biolabs, Catalog \#E7120S) (Liu et al., 2019; Vaisvila et al., 2019; Sun et al., 2021a). Then the converted DNA was tagged with Illumina compatible adapter and amplified to an appropriate library concentration using Accel-NGS ${ }^{\circledR}$ Methyl-Seq DNA Library Kit (Swift BioSciences) coupled with NEB Next ${ }^{\circledR}$ Multiplex Oligos for Illumina ${ }^{\circledR}$ (New England Biolabs). Libraries were checked for quality and quantified using Agarose Gel Electrophoresis and Qubit3.0 individually. 


\section{Sequence Alignment and Peak Identification}

Sequencing reads were trimmed by trimmomatic with the trim tail option set true and mapped to the mouse genome (mm10) by bowtie2. The peak analysis was conducted by MACS2 call peaks, under the paired-end mode with bam file as input format. Afterward, the peaks were annotated by HOMER annotate Peak, and mm10 was used as the reference (Supplementary Table S1).

\section{Single-Base Resolution Analysis}

The numbers as well as site information of converted and unconverted cytosines in samples were recognized by the software Bismark, with the criterion that the C site cutoff is greater than zero; that is, the conversion was valid as long as any read indicates modification of the site. The process of running Bismark can be split into three steps. First, conduct bisulfite conversion on the genome of interest and build its index to allow bowtie2 alignments. Second, run Bismark alignment step, which means the actual bisulfite alignment and methylation calling part. Finally, extract the methylation information from the Bismark alignment output (Supplementary Table S2). Validation of genomic $5 \mathrm{hmC}$ at the $\mathrm{CH}$ sites was done on AbaSI-digested glucosylated ESC genomic DNA followed by real-time PCR (Sun et al., 2013; Sun et al., 2021b). Real-time PCR of digested DNA was done with iQ SYBR Green Supermix (Bio-Rad Laboratories, Hercules, CA, USA: 170-8882). All real-time PCR primers and the method are listed in Supplementary Table $\mathbf{S} 3$ for $\mathrm{CH}$ sites.

\section{Definition of Enhancer Subgroups and Motif Analysis}

Enhancers with evidence showing their interaction with distal genomic regions were considered as the most active enhancer subgroup (interacting enhancers). The genomic locations of these enhancers were obtained from published ChIA-PET dataset. The active and poised enhancers were defined by using histone modification markers. Both H3K4me1- and H3K27ac-enriched regions were obtained from previous publication. Liftover was used to convert the genomic location from $\mathrm{mm} 8$ to $\mathrm{mm} 10$. Regions that overlapped with interacting enhancers were discarded. Regions enriched with H3K27ac were considered as active enhancers. Regions only enriched with H3K4me1 but not $\mathrm{H} 3 \mathrm{~K} 27 \mathrm{ac}$ were considered as poised enhancers. To detect TF motifs around $5 \mathrm{hmC}$-modified cytosines, we performed de novo motif analysis with HOMER find Motifs Genome around the $5 \mathrm{hmC}$ sites in mESCs.

\section{RESULTS}

\section{DIP-CAB-Seq Can Generate Deaminated DNA Library From the Pull-Down DNA Fragments}

We present here a selective chemical labeling coupled with bisulfite-free sequencing (DIP-CAB-Seq) approach to generate the genome-wide, single-base resolution maps for $5 \mathrm{hmC}$. In the current approach, we first selectively labeled and enriched $5 \mathrm{hmC}$ containing DNA fragments by using the glucosyltransferase and azido-UDP-glucose-based DNA profiling prior to the subsequent deamination (Figure 1). We then performed the deamination on the enriched fragments by using deaminase-based bisulfite-free reaction. The normal cytosine and $5 \mathrm{mC}$ can be deaminated, while $5 \mathrm{hmC}$ remained as glucosylated $5 \mathrm{hmC}\left(\mathrm{N}_{3}-5 \mathrm{gmC}\right)$. Therefore, $5 \mathrm{hmC}$ will be read as $\mathrm{C}$ after PCR amplification, while normal C and $5 \mathrm{mC}$ will be read as $\mathrm{T}$. After the single-stranded library construction and PCR amplification, the $5 \mathrm{hmC}$ containing fragments will be selectively amplified, while the $5 \mathrm{hmC}$ can be read in single-base resolution and generate the precise genomic locations of $5 \mathrm{hmC}$.

The first challenge is whether we can generate high-quality next-generation sequencing library from the trace amount pull-down DNA. We validated that approach by generating $5 \mathrm{hmC}$ library in genomic DNA of mECS. We constructed ACE-Seq libraries and DIP-CAB-Seq libraries from two replicated mECS gDNA. The output data size was determined by library input. After getting the DNA library, we examined its concentration used Qubit. The first normalization was conducted through dilution between library's concentration and validated by quantitative PCR. The second normalization was conducted base on the quantitative-PCR data. A library mixture was prepared by pooling the same amount of diluted library. The library mixture was sequenced on Nestseq500 device by following the manufacturer's instruction. We observed $99.89 \%$ deamination of control DNA. The ACE-Seq library generated $35,067,558$ reads with $68 \%$ mapping ratio, while the DIP-CAB-Seq library generated $32,769,029$ reads with $61 \%$ mapping ratio, not far from the former result. The DIP-CABSeq methods have slightly lower mapping ratio than ACE-Seq method, which is fully acceptable considering the ultra-low input DNA amount in the library construction step in DIPCAB-Seq method. Both DIP-CAB-Seq libraries and regular ACE-Seq libraries have similar total sequencing reads, which guaranteed us a fair comparison between the ability of the two methods in detecting the $5 \mathrm{hmC}$ sites in the genome. These data indicate that we can successfully construct the $5 \mathrm{hmC}$ singlebase resolution library from the enriched DNA fragments.

\section{DIP-CAB-Seq Can Selectively Enrich 5-Hydroxymethylcytosine-Containing DNA Fragments}

Using the DIP-CAB-Seq strategy, we performed both regular $\mathrm{hmC}$-Seal profiling and single-base resolution mapping of $5 \mathrm{hmC}$ on mESC genomic DNA. The profiling analysis indicated that $5 \mathrm{hmC}$ from both libraries accumulate at the intergenic and intron regions in mESCs (Figure 2). This observation is consistent with previous findings (Song et al., 2011). We barely observed any abnormal 5hmC signals, nor did we observe any noticeable changes of $5 \mathrm{hmC}$ at these genomic element regions between the two methods. Therefore, the DIP-CAB-Seq can totally inherit 


\section{นำ}

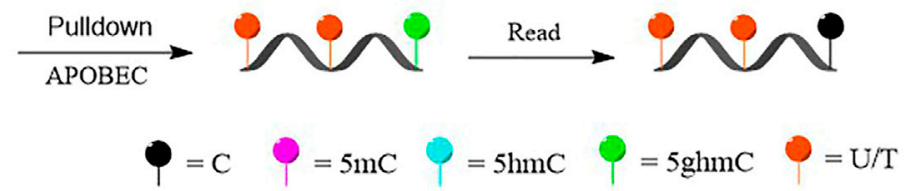

FIGURE 1 | Schematic diagram of selectively labeling and deaminase-based sequencing of 5-hydroxymethylcytosine (5hmC) in DNA (DIP-CAB-Seq).

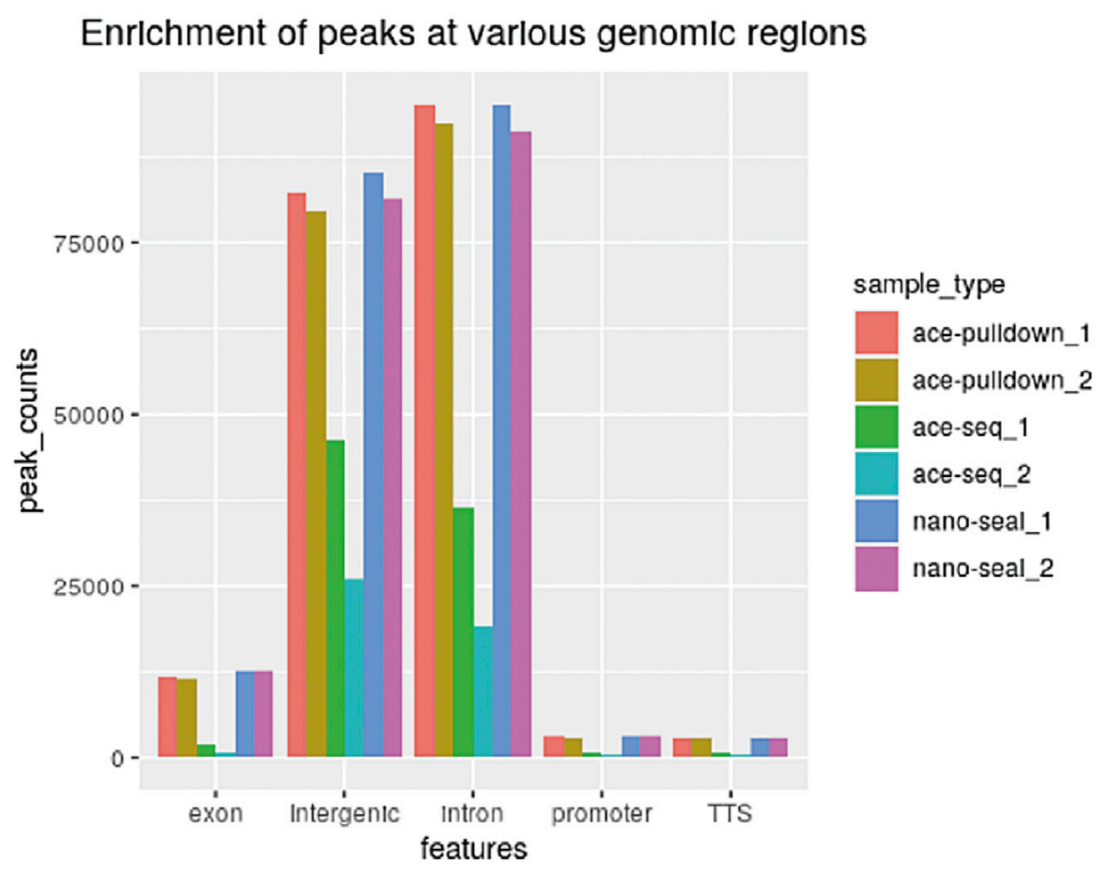

FIGURE 2 | Enrichment of peaks at various genomic regions (ACE-pulldown: DIP-CAB-Seq method).

the profiling information that the traditional hmC-Seal method carried.

\section{DIP-CAB-Seq Can Detect Single-Base Information With Limited Sequencing Depth}

We next analyzed $5 \mathrm{hmC}$ enriched regions using the base-resolution data in ACE-Seq and DIP-CAB-Seq so as to evaluate the repeatability of both methods. We observed 5,936,200 $5 \mathrm{hmC}$ sites in CpG context in the union set of two ACE-Seq samples, while there are only $291,5275 \mathrm{hmC}$ sites, that is, only $4.9 \%$ of the union set in the intersection set of both samples. On the other hand, we found $7,821,4535 \mathrm{hmC}$ sites in the CpG context in the union set of the DIPCAB-Seq samples, and there are $1,273,9535 \mathrm{hmC}$ sites in the intersection set of both samples, accounting for $16.3 \%$ of the union set. These data indicate that the DIP-CAB-Seq method has higher detection ability and consistency than ACE-Seq with the same sequencing depth. Considering that the total sequencing depth is much lower than that of a regular expensive whole-genome $5 \mathrm{hmC}$ sequencing, the DIP-CAB-Seq method shows much better application potential in large-scale sample studies. We also detect $5 \mathrm{hmC}$ signals in $\mathrm{CHH}$ and $\mathrm{CHG}$, which may have $5 \mathrm{hmC}$ sites according to the previous study. As expected, DIP-CAB-Seq methods showed higher consistency than ACE-Seq. We observed many $\mathrm{CHH}$ and $\mathrm{CHG}$ sites from the ACE-Seq library, which may come from the false-positive signal of the ACE-Seq due to the nonperfect deamination. The amount of $5 \mathrm{hmC}$ sites in $\mathrm{CHH}$ and $\mathrm{CHG}$ is shown in the figure below (Table 1). Therefore, the enrichment of the $5 \mathrm{hmC}$ fragments can also decrease the false-positive signals even if the deamination reagents are not perfect in a bisulfite-free system. 
TABLE 1 | The amount of $5 \mathrm{hmC}$ sites in $\mathrm{CHH}$ and $\mathrm{CHG}$.

\begin{tabular}{llccc}
\hline Methods & Sample_num & CpG & CHG & CHH \\
\hline ACE-Seq & ACE_1 & $6,342,475$ & $20,163,184$ & $62,652,700$ \\
ACE-Seq & ACE_2 & $1,183,130$ & $3,235,097$ & $11,574,624$ \\
DIP-CAB-Seq & DIP-CAB_1 & $5,427,793$ & $1,418,759$ & $4,081,655$ \\
DIP-CAB-Seq & DIP-CAB_2 & $5,818,168$ & $2,586,082$ & $7,293,592$
\end{tabular}

Note. 5hmC, 5-hydroxymethylcytosine.

We further analyzed the single-base resolution signals from both methods in genomic elements. The following four pie charts (Figure 3) show the distribution of $5 \mathrm{hmC}$ sites in samples of ACE-Seq and DIP-CAB-Seq in different feature regions. The proportions are quite different between samples of ACE-Seq, while those of samples of DIP-CAB-Seq are almost the same.

Based on the above findings, we observed much better consistency from DIP-CAB-Seq method than ACE-Seq, indicating the DIPCAB-Seq method could have better understanding of the biology with limited sequencing depth (Figure 4).

\section{DIP-CAB-Seq Can Detect Detailed Preferential Occurrences of 5-Hydroxymethylcytosine}

With single-base resolution information available, we continued to investigate the detailed preferential occurrences of $5 \mathrm{hmC}$ sites in the genome to understand the potential of this method in biology study.

The previous base-resolution mapping of $5 \mathrm{hmC}$ allowed for the determination of the surrounding base composition of $5 \mathrm{hmC}$ sites in mESCs (Ring et al., 2017). We aligned our 5hmC sites in CG context and examined the base compositions (Figure 5). Compared with reported results, our data possess a similar local sequence context, having increased guanine abundance with a depletion of thymine.

We then performed motif analysis with HOMER32 at \pm 100 bp region around the $5 \mathrm{hmC}$ sites. We studied the well-known 5hmC containing motif Klf4, Oct4, Hifla, Esrrb, and Sox2; our motif analysis successfully identified these motifs around the $5 \mathrm{hmC}$ sites in mESCs (Figure 6). Our data indicated that our method can obtain these detailed $5 \mathrm{hmC}$ signals with very low sequencing depth as compared with the reported method.

\section{DISCUSSION}

The current dilemma between cost and sequencing resolution has hampered many biological and clinical studies of $5 \mathrm{hmC}$. Generally, $5 \mathrm{hmC}$ has 10 times lower genome percentage than $5 \mathrm{mC}$, and $5 \mathrm{hmC}$ is distributed broadly across the genome, which requires very deep sequencing for detecting low abundant $5 \mathrm{hmC}$ sites. Although the profiling methods provide a cheap manner to map the $5 \mathrm{hmC}$ peak, it is much more difficult to quantify the $5 \mathrm{hmC}$ signals than the single-

\section{ace1}

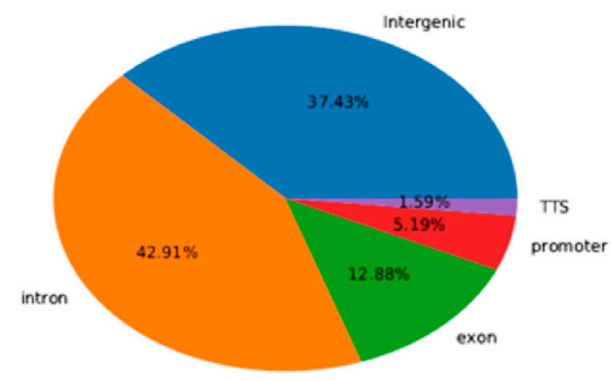

pulldown1

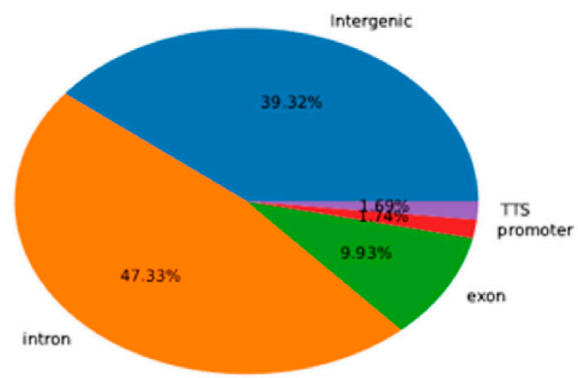

ace2

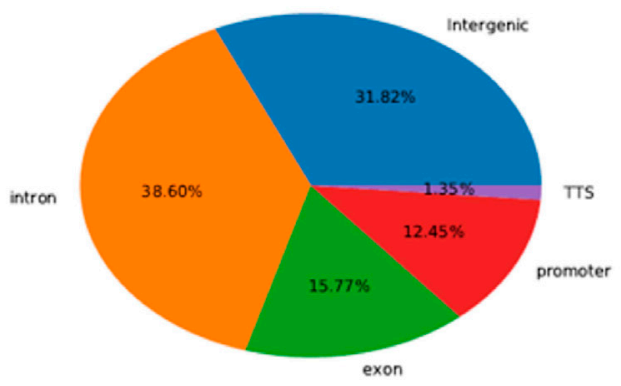

pulldown2

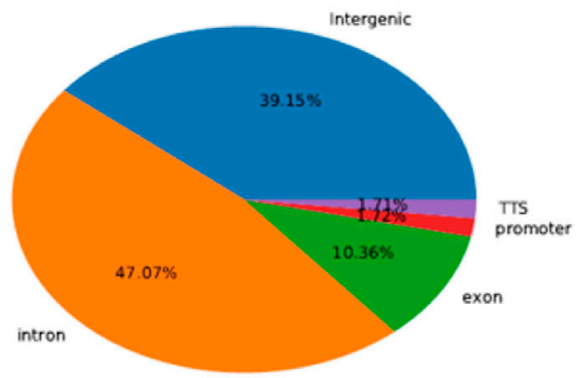

FIGURE 3 | The proportion of 5-hydroxymethylcytosine (5hmC) in various areas 
ace_pulldown 5hmc sites in $\mathrm{CpG}$

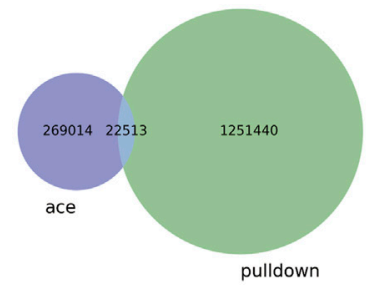

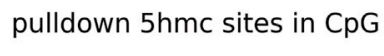

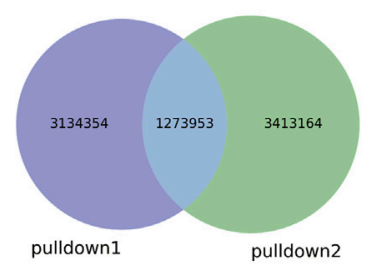

ace $5 \mathrm{hmc}$ sites in $\mathrm{CpG}$

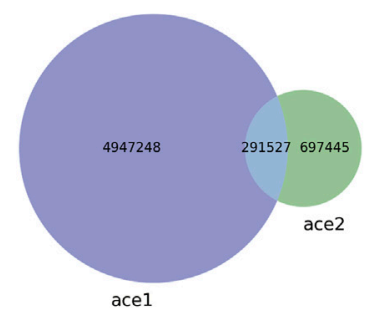

FIGURE 4 | Repeatability of 5-hydroxymethylcytosine (5hmC) sites with ACE, DIP-CAB-Seq, and Nano-seal methods.

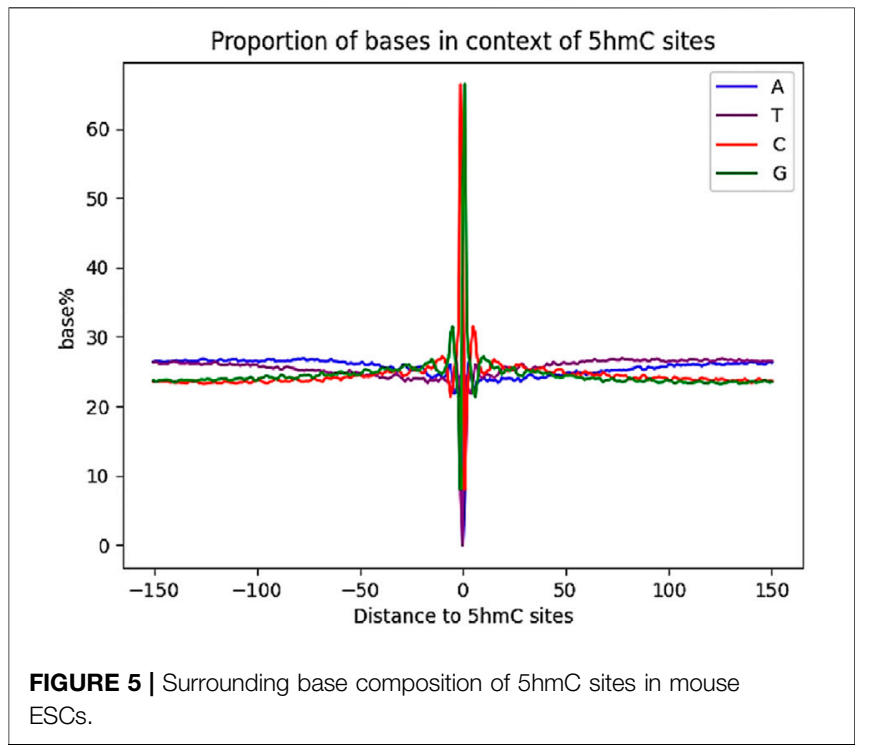

\begin{tabular}{llcc|} 
Name & $\begin{array}{c}\text { SEQ19588 } \\
\text { P-value }\end{array}$ & $\begin{array}{c}\text { SEQ19589 } \\
\text { P-value }\end{array}$ \\
& Hif1b & $1 \mathrm{e}-16$ & $1 \mathrm{e}-14$ \\
Figure 6 I Motif analysis of 5-hydroxymethylcytosine (5hmC) sites in \\
mouse embryonic stem cells (ESCs).
\end{tabular}

base resolution method. The DIP-CAB-Seq method we developed can solve this problem; although we cannot quantify the $5 \mathrm{hmC}$ abundance for a specific $5 \mathrm{hmC}$ site, it provides much better resolution than the regular profiling method. We showed that the base-resolution data from the very limited sequencing depth can provide detailed $5 \mathrm{hmC}$ loci information. It is well known that $5 \mathrm{hmC}$ profiling has the potential to be a clinical tool in different clinical questions. The current profiling can only calculate the $5 \mathrm{hmC}$ score based on $5 \mathrm{hmC}$ read density, which needs much adjustment between the samples and cohorts. To further study the role of $5 \mathrm{hmC}$ in clinical application, our method can provide better quantification information and could be more reliable than the profiling method.

\section{CONCLUSION}

Herein, we developed a method based on ESC whole-genome analysis, which could enrich $5 \mathrm{hmC}$-containing DNA by selective chemical labeling and locate $5 \mathrm{hmC}$ sites at single-base resolution with enzyme-based deamination. The combination experimental design is an extension of previous methods, and we hope that this cost-effective single-base resolution $5 \mathrm{hmC}$ sequencing method could be used to promote the mechanism and diagnostic research of $5 \mathrm{hmC}$.

\section{DATA AVAILABILITY STATEMENT}

The original contributions presented in the study are publicly available. These data can be found here: National Center for Biotechnology Information (NCBI) BioProject database under accession number PRJNA732757.

\section{AUTHOR CONTRIBUTIONS}

I) Conception and design: XL. II) Administrative support: YX. III) Provision of study materials: WG and XL. IV) Collection and assembly of data: XL, XS, and YG. V) Data analysis and interpretation: XL and XS. VI) Article writing: all authors. VII) Final approval of article: all authors.

\section{FUNDING}

This research was supported by the Beijing Natural Science Foundation (7194312 to XL), Beijing Key Clinical Specialty for Laboratory Medicine-Excellent Project (No. ZK201000), 
National Key Research and Development Program of China (2017YFC1601502) and Beijing Municipal Science and Technology Project (Z181100001618015), and Beijing Key Laboratory for Mechanisms Research and Precision Diagnosis of Invasive Fungal Diseases.

\section{REFERENCES}

Colquitt, B. M., Allen, W. E., Barnea, G., and Lomvardas, S. (2013). Alteration of Genic 5-hydroxymethylcytosine Patterning in Olfactory Neurons Correlates with Changes in Gene Expression and Cell Identity. Proc. Natl. Acad. Sci. USA 110, 14682-14687. doi:10.1073/pnas.1302759110

Cui, L., Chung, T. H., Tan, D., Sun, X., and Jia, X.-Y. (2014). JBP1-seq: a Fast and Efficient Method for Genome-wide Profiling of 5hmC. Genomics 104, 368-375. doi:10.1016/j.ygeno.2014.08.023

Gibas, P., Narmontė, M., Staševskij, Z., Gordevičius, J., Klimašauskas, S., and Kriukienè, E. (2020). Precise Genomic Mapping of 5-hydroxymethylcytosine via Covalent Tether-Directed Sequencing. Plos Biol. 18 (4), e3000684. doi:10.1371/journal.pbio.3000684

Guo, W., Wang, S., Zhang, X., Shi, M., Duan, F., Hao, J., et al. (2021). Acidic pH Transiently Prevents the Silencing of Self-Renewal and Dampens MicroRNA Function in Embryonic Stem Cells. Sci. Bull. 66, 1319-1329. doi:10.1016/ j.scib.2021.03.005

Han, D., Lu, X., Shih, A. H., Nie, J., You, Q., Xu, M. M., et al. (2016). A Highly Sensitive and Robust Method for Genome-wide 5hmC Profiling of Rare Cell Populations. Mol. Cel 63, 711-719. doi:10.1016/j.molcel.2016.06.028

Hu, L., Liu, Y., Han, S., Yang, L., Cui, X., Gao, Y., et al. (2019). Jump-seq: Genomewide Capture and Amplification of 5-Hydroxymethylcytosine Sites. J. Am. Chem. Soc. 141 (22), 8694-8697. doi:10.1021/jacs.9b02512

Kriaucionis, S., and Heintz, N. (2009). The Nuclear DNA Base 5hydroxymethylcytosine Is Present in Purkinje Neurons and the Brain. Science 324, 929-930. doi:10.1126/science.1169786

Liu, Y., Siejka-Zielińska, P., Velikova, G., Bi, Y., Yuan, F., Tomkova, M., et al. (2019). Bisulfite-free Direct Detection of 5-methylcytosine and 5-hydroxymethylcytosine at Base Resolution. Nat. Biotechnol. 37 (4), 424-429. doi:10.1038/s41587-019-0041-2

Mellén, M., Ayata, P., Dewell, S., Kriaucionis, S., and Heintz, N. (2012). MeCP2 Binds to $5 \mathrm{hmC}$ Enriched within Active Genes and Accessible Chromatin in the Nervous System. Cell 151, 1417-1430. doi:10.1016/j.cell.2012.11.022

Pastor, W. A., Huang, Y., Henderson, H. R., Agarwal, S., and Rao, A. (2012). The GLIB Technique for Genome-wide Mapping of 5-hydroxymethylcytosine. Nat. Protoc. 7, 1909-1917. doi:10.1038/nprot.2012.104

Petterson, A., Chung, T. H., Tan, D., Sun, X., and Jia, X.-Y. (2014). RRHP: a TagBased Approach for 5-hydroxymethylcytosine Mapping at Single-Site Resolution. Genome Biol. 15, 456. doi:10.1186/s13059-014-0456-5

Ring, J. D., Sturk-Andreaggi, K., Peck, M. A., and Marshall, C. (2017). A Performance Evaluation of Nextera XT and KAPA HyperPlus for Rapid Illumina Library Preparation of Long-Range Mitogenome Amplicons. Forensic Sci. Int. Genet. 29, 174-180. doi:10.1016/j.fsigen.2017.04.003

Song, C.-X., Szulwach, K. E., Fu, Y., Dai, Q., Yi, C., Li, X., et al. (2011). Selective Chemical Labeling Reveals the Genome-wide Distribution of 5hydroxymethylcytosine. Nat. Biotechnol. 29, 68-72. doi:10.1038/nbt.1732

Sun, Z., Dai, N., Borgaro, J. G., Quimby, A., Sun, D., Corrêa, I. R., et al. (2015). A Sensitive Approach to Map Genome-wide 5-hydroxymethylcytosine and 5-formylcytosine at Single-Base Resolution. Mol. Cel 57, 750-761. doi:10.1016/j.molcel.2014.12.035

Sun, Z., Terragni, J., Borgaro, J. G., Liu, Y., Yu, L., Guan, S., et al. (2013). Highresolution Enzymatic Mapping of Genomic 5-hydroxymethylcytosine in Mouse Embryonic Stem Cells. Cel Rep. 3 (2), 567-576. doi:10.1016/j.celrep.2013.01.001

Sun, Z., Terragni, J., Zhu, Z., Zheng, Y., and Pradhan, S. (2021). : High-Resolution Enzymatic Mapping of Genomic 5-Hydroxymethylcytosine. Methods Mol. Biol. 2272, 13-27. doi:10.1007/978-1-0716-1294-1_2

\section{SUPPLEMENTARY MATERIAL}

The Supplementary Material for this article can be found online at: https://www.frontiersin.org/articles/10.3389/fgene.2021.749211/ full\#supplementary-material

Sun, Z., Vaisvila, R., Hussong, L.-M., Yan, B., Baum, C., Saleh, L., et al. (2021). Nondestructive Enzymatic Deamination Enables Single-Molecule Long-Read Amplicon Sequencing for the Determination of 5-methylcytosine and 5hydroxymethylcytosine at Single-Base Resolution. Genome Res. 31 (2), 291-300. doi:10.1101/gr.265306.120

Szulwach, K. E., Li, X., Li, Y., Song, C.-X., Wu, H., Dai, Q., et al. (2011). 5hmC-mediated Epigenetic Dynamics during Postnatal Neurodevelopment and Aging. Nat. Neurosci. 14, 1607-1616. doi:10.1038/nn.2959

Tahiliani, M., Koh, K. P., Shen, Y., Pastor, W. A., Bandukwala, H., Brudno, Y., et al. (2009). Conversion of 5-methylcytosine to 5-hydroxymethylcytosine in Mammalian DNA by MLL Partner TET1. Science 324, 930-935. doi:10.1126/science.1170116

Tan, L., Xiong, L., Xu, W., Wu, F., Huang, N., Xu, Y., et al. (2013). Genome-wide Comparison of DNA Hydroxymethylation in Mouse Embryonic Stem Cells and Neural Progenitor Cells by a New Comparative hMeDIP-Seq Method. Nucleic Acids Res. 41, e84. doi:10.1093/nar/gkt091

Taylor, S. E., Li, Y. H., Smeriglio, P., Rath, M., Wong, W. H., and Bhutani, N. (2016). Stable 5-Hydroxymethylcytosine (5hmC) Acquisition Marks Gene Activation during Chondrogenic Differentiation. J. Bone Miner. Res. 31, 524-534. doi:10.1002/jbmr.2711

Thomson, J. P., Hunter, J. M., Nestor, C. E., Dunican, D. S., Terranova, R., Moggs, J. G., et al. (2013). Comparative Analysis of Affinity-Based 5hydroxymethylation Enrichment Techniques. Nucleic Acids Res. Techniques. Nucleic Acids Res. 41, e206. doi:10.1093/nar/gkt1080

Vaisvila, R., Ponnaluri, V. K. C., Sun, Z., Langhorst, B. W., Saleh, L, Guan, S., et al. (2019). EM-seq: Detection of DNA Methylation at Single Base Resolution from Picograms of DNA. bioRxiv 12, doi:10.1101/ 2019.12.20.884692

Wang, T., Loo, C. E., and Kohli, R. M. (2021). Enzymatic Approaches for Profiling Cytosine Methylation and Hydroxymethylation. Mol. Metab. 8, 101314. doi:10.1016/j.molmet.2021.101314

Wray, J., Kalkan, T., Gomez-Lopez, S., Eckardt, D., Cook, A., Kemler, R., et al. (2011). Inhibition of Glycogen Synthase Kinase-3 Alleviates Tcf3 Repression of the Pluripotency Network and Increases Embryonic Stem Cell Resistance to Differentiation. Nat. Cel Biol. 13, 838-845. doi:10.1038/ ncb2267

Conflict of Interest: The authors declare that the research was conducted in the absence of any commercial or financial relationships that could be construed as a potential conflict of interest.

Publisher's Note: All claims expressed in this article are solely those of the authors and do not necessarily represent those of their affiliated organizations, or those of the publisher, the editors, and the reviewers. Any product that may be evaluated in this article, or claim that may be made by its manufacturer, is not guaranteed or endorsed by the publisher.

Copyright (C) $2021 \mathrm{Li}$, Shi, Gong, Guo, Liu, Peng and Xu. This is an open-access article distributed under the terms of the Creative Commons Attribution License (CC BY). The use, distribution or reproduction in other forums is permitted, provided the original author(s) and the copyright owner(s) are credited and that the original publication in this journal is cited, in accordance with accepted academic practice. No use, distribution or reproduction is permitted which does not comply with these terms. 